\title{
Crescimento do milho em resposta à rizobactérias, molibdênio $e$ nitrogênio
}

Bactérias como Azospirillum brasilense e Rhizobium tropici apresentam potencial para melhorar o desempenho agronômico do milho em função de sua atividade diazotrófica e, também por atuarem como rizobactérias promotoras do crescimento de plantas. Além disso, o uso destas espécies microbianas pode ampliar os benefícios isolados e combinados do molibdênio e nitrogênio aplicados por ocasião da semeadura. $O$ objetivo deste trabalho foi avaliar os efeitos isolados e combinados de Azospirillum brasilense, Rhizobium tropici, molibdênio e nitrogênio no crescimento do milho AG 1051. O experimento foi conduzido em casa de vegetação seguindo-se o delineamento inteiramente casualizado, em esquema fatorial $2 \times 2 \times 2 \times 2$, com 5 repetições, considerando a ausência e presença de Azospirillum brasilense, Rhizobium tropici e molibdênio nas sementes e nitrogênio no solo. No pendoamento foi avaliada a altura da planta, diâmetro basal do colmo, o nitrogênio acumulado na parte aérea e as massas secas da parte aérea, raízes e total. Foi observado maior crescimento do milho devido à adubação nitrogenada. Também se verificou que a inoculação de Azospirillum brasilense nas sementes potencializou o efeito da adubação nitrogenada em incrementar a quantidade de nitrogênio acumulada na parte aérea das plantas. Além disso, esta variável foi aumentada devido à coinoculação das rizobactérias associada à adubação molíbdica.

Palavras-chave: Zea mays; Microrganismos diazotróficos; RPCPs; Coinoculação; Fertilizantes químicos.

\section{Maize growth in response to rhizobacteria, molybdenum and nitrogen}

\begin{abstract}
Bacteria such as Azospirillum brasilense and Rhizobium tropici have the potential to improve the agronomic performance of maize due to its diazotrophic activity and also to act as plant growth promoting rhizobacteria. In addition, the use of these microbial species may enhance the isolated and combined benefits of molybdenum and nitrogen applied at sowing. The objective of this work was to evaluate the isolated and combined effects of Azospirillum brasilense, Rhizobium tropici, molybdenum and nitrogen on the growth of AG 1051 maize. The experiment was carried out in a greenhouse following a completely randomized design in a $2 \times 2 \times 2 \times 2$ factorial scheme with 5 repetitions, considering the absence and presence of Azospirillum brasilense, Rhizobium tropici and molybdenum in the seeds and nitrogen in the soil. In the tearing, the plant height, basal diameter of the stem, the accumulated nitrogen in the aerial part and the dry mass of the aerial part, roots and total were evaluated. Higher maize growth was observed due to nitrogen fertilization. It was also verified that the inoculation of Azospirillum brasilense in the seeds potentiated the effect of nitrogen fertilization in increasing the amount of accumulated nitrogen in the aerial part of the plants. In addition, this variable was increased due to the co-inoculation of rhizobacteria associated with molybdic fertilization.
\end{abstract}

Keywords: Zea mays; Diazotrophic microorganisms; PGPR; Co-inoculation; Chemical fertilizers.

Topic: Desenvolvimento, Sustentabilidade e Meio Ambiente

Reviewed anonymously in the process of blind peer
Received: $14 / 06 / 2019$

Approved: $15 / 07 / 2019$

\section{Angelita Aparecida Coutinho Picazevicz (it) Instituto Federal de Rondônia, Brasil http://lattes.cnpq.br/9808078161906025 http://orcid.org/0000-0001-9570-2942 angelitaacoutinho@gmail.com \\ Jorge Ferreira Kusdra (D) \\ Universidade Federal do Acre, Brasil \\ http://lattes.cnpq.br/1089099197793431 \\ http://orcid.org/0000-0003-3651-468X \\ kusdra@globo.com \\ Andréia de Lima Moreno (iD \\ Universidade Federal do Acre, Brasil \\ http://lattes.cnpq.br/4252988221826496 \\ http://orcid.org/0000-0002-5406-5542 \\ andreiatantalo.lider@gmail.com}

\section{Referencing this:}

PICAZEVICZ, A. A. C.; KUSDRA, J. F.; MORENO, A. L. Crescimento do milho em resposta à rizobactérias, molibdênio e nitrogênio. Revista Ibero-Americana de Ciências Ambientais, v.10, n.4, p.167-174, 2019. DOI: http://doi.org/10.6008/CBPC2179-6858.2019.004.0013 


\section{INTRODUÇÃO}

Bactérias diazotróficas como Azospirillum brasilense, já recomendadas para o milho, podem contribuir por meio da fixação biológica do nitrogênio (FBN) e pela atividade como rizobactérias promotoras do crescimento de plantas $\left(\mathrm{RPCP}_{\mathrm{s}}\right)$ para reduzir a aplicação de grandes quantidades de adubo nitrogenado sintético, diminuir a necessidade de adubações em cobertura, aumentar a disponibilidade do elemento para as plantas, bem como melhorar o desempenho agronômico da cultura (ARAÚJO et al., 2014; COSTA et al., 2015; MORAIS et al., 2016; QUADROS et al., 2014). Além disso, outras bactérias fixadoras de nitrogênio, como os rizóbios, indicados normalmente para o cultivo de fabáceas nodulíferas, podem também atuar como RPCPs em espécies de poáceas (SINGH et al., 2013; TAN et al., 2014). Neste sentido, o uso de inoculantes rizobianos no milho pode contribuir tanto para aumentar o crescimento vegetal quanto otimizar o aproveitamento pelas plantas do nitrogênio aplicado via adubação (DARTORA et al., 2016; OSÓRIO FILHO et al., 2016).

Em razão dos múltiplos mecanismos de ação das RPCPs (produção de fitohormônios vegetais, solubilização de nutrientes, controle de fitopatógenos) é desejável o uso simultâneo de duas ou mais espécies nas culturas visando potencializar seus efeitos isolados e ampliar os benefícios da FBN, otimizar o aproveitamento pelas plantas de nutrientes adicionados ao solo e melhorar o desempenho agronômico das culturas (NOGUEIRA \& HUNGRIA, 2013). Mazzuchelli et al. (2014) observaram que, no milho cultivado em solo sem adubação nitrogenada, a coinoculação de Bacillus subtilis e Azospirillum brasilense propiciou incremento na altura das plantas e na produtividade dos grãos. Resultados positivos para o crescimento do milho derivados do uso combinado de RPCPs foram obtidos também por Akhtar et al. (2014) ao verificarem aumento da altura, diâmetro de colmo, massa seca de parte aérea e das quantidades de fósforo e nitrogênio acumuladas nas plantas em resposta a aplicação simultânea de Rhizobium sp. e Bacillus sp. nas sementes e de nitrogênio $\left(120 \mathrm{~kg} \mathrm{ha}^{-1}\right)$ e fósforo $\left(60 \mathrm{~kg} \mathrm{ha}^{-1}\right)$ no solo por ocasião da semeadura.

No cultivo do milho, o uso de molibdênio pode contribuir para melhorar o aproveitamento pelas plantas do nitrogênio adicionado ao solo via adubação (VALENTINI et al., 2005). A aplicação combinada deste micronutriente com rizobactérias pode também potencializar a atividade diazotrófica destes organismos em poáceas e ampliar a quantidade de nitrogênio acumulada nestas (GANAPATHY \& SAVALGI, 2006). Este benefício deriva do fato do molibdênio participar da composição e ativação das enzimas nitrogenase, envolvida na FBN e redutase do nitrato, que viabiliza o aproveitamento de nitrogênio pelas plantas via solo.

Considerando que com a coinoculação de rizobactérias combinada à adubação molíbdica e nitrogenada na semeadura os benefícios para as plantas derivados do uso destas fontes podem ser ampliados e o desempenho agronômico da cultura melhorado, objetivou-se com esta pesquisa avaliar a magnitude do efeito isolado e combinado de Azospirillum brasilense, Rhizobium tropici, molibdênio e nitrogênio no crescimento do milho AG 1051. 


\section{MATERIAL E MÉTODOS}

O experimento com milho híbrido duplo AG 1051 foi conduzido em casa de vegetação localizada na área experimental do Instituto Federal de Rondônia, unidade de Cacoal, Rondônia ( $11^{\circ} 28^{\prime} \mathrm{S} ; 61^{\circ} 22^{\prime}$ O; $184 \mathrm{~m}$ ), no período de maio a julho de 2016, seguindo o delineamento inteiramente casualizado em arranjo fatorial $2 \times 2 \times 2 \times 2$, cujos fatores corresponderam à ausência e presença de Azospirillum brasilense, Rhizobium tropici, molibdênio nas sementes e nitrogênio no solo. Considerou-se para cada um dos 16 tratamentos combinados 5 repetições, totalizando 80 unidades experimentais, as quais constituíram-se de vasos de policloreto de polivinila (PVC) com diâmetro de $200 \mathrm{~mm}$, altura de $50 \mathrm{~cm}$ e volume de 15,7 dm .

O solo utilizado como substrato foi retirado da camada superficial $(0-20 \mathrm{~cm})$ de uma área em pousio e antes de ser adicionado aos vasos elevou-se seu nível de fertilidade mediante adubação fosfatada com 100 $\mathrm{mg}$ de $\mathrm{P}$ na forma de superfosfato simples $\left(18 \%\right.$ de $\left.\mathrm{P}_{2} \mathrm{O}_{5}\right)$ e potássica com $100 \mathrm{mg}$ de $\mathrm{K}$ na forma de cloreto de potássio $\left(58 \%\right.$ de $\left.\mathrm{K}_{2} \mathrm{O}\right)$ quinze dias antes da semeadura. Na instalação do experimento as características químicas e físicas do solo, determinadas de acordo com EMBRAPA (1997), foram: $\mathrm{pH}\left(\mathrm{CaCl}_{3}\right)=5,5$; matéria orgânica $=35,8 \mathrm{~g} \cdot \mathrm{dm}^{-3} ; \mathrm{P}=15,1 \mathrm{mg} \cdot \mathrm{dm}^{-3} ; \mathrm{K}=96,4 \mathrm{mg} \cdot \mathrm{dm}^{-3} ; \mathrm{Ca}=3,3 \mathrm{cmol}_{\mathrm{c}} \cdot \mathrm{dm}^{-3} ; \mathrm{Mg}=1,2 \mathrm{cmol}_{\mathrm{c}} \cdot \mathrm{dm}^{-3} ; \mathrm{Al}=0,0$ $\mathrm{cmol}_{\mathrm{c}} \cdot \mathrm{dm}^{-3} ; \mathrm{H}+\mathrm{Al}=3,5 \mathrm{cmol}_{\mathrm{c}} \cdot \mathrm{dm}^{-3} ;$ soma de bases $=4,7 \mathrm{cmol}_{\mathrm{c}} \cdot \mathrm{dm}^{-3} ; \mathrm{CTC}=8,2 \mathrm{cmol}_{\mathrm{c}} \mathrm{dm}^{-3} ;$ saturação por bases $=$ $57 \%$; areia $=523 \mathrm{~g} . \mathrm{kg}^{-1} ;$ silte $=117 \mathrm{~g} \cdot \mathrm{kg}^{-1} ;$ argila $=360 \mathrm{~g} \cdot \mathrm{kg}^{-1} ;$ densidade aparente $=1,5 \mathrm{~g} \cdot \mathrm{cm}^{-3}$; densidade de partículas $=2,4 \mathrm{~g} \mathrm{~cm}^{-3}$ e porosidade total $=37 \%$.

As fontes das rizobactérias utilizadas foram inoculantes comerciais turfosos à base de Azospirillum brasilense estirpes AbV5 e AbV6 e Rhizobium tropici SEMIA 4077 e SEMIA 4088. O número mais provável de células viáveis dos produtos biológicos foi estimado pelo método de diluição seriada e contagem de colônias em placas (TORTORA et al., 2017) contendo os meios seletivos para Azospirillum [ácido málico (5 g), fosfato dipotássico $(0,5 \mathrm{~g})$, sulfatos de ferro $(0,5 \mathrm{~g})$, manganês $(0,01 \mathrm{~g})$ e magnésio $(0,2 \mathrm{~g})$, cloretos de sódio $(0,1 \mathrm{~g})$ e de cálcio $(0,02 \mathrm{~g})$, molibdato de sódio $(0,002 \mathrm{~g})$, azul de bromotimol $(0,002 \mathrm{~g})$, ágar $(20 \mathrm{~g})$, hidróxido de potássio (4 g) e água destilada (1000 mL)] e Rhizobium [manitol (10 g), extrato de levedura $(1 \mathrm{~g})$, cloreto de sódio $(0,1 \mathrm{~g})$, sulfato de magnésio $(0,2 \mathrm{~g})$, fosfato dipotássico $(0,5 \mathrm{~g})$ ágar $(20 \mathrm{~g})$ e água destilada $(1000 \mathrm{~mL})$ ]. A quantidade de células viáveis dos microrganismos estimadas por grama de inoculante foi de $1,3 \times 10^{9}$ para Azospirillum brasilense e de $1,8 \times 10^{9}$ para Rhizobium tropici. Para molibdênio e nitrogênio, utilizou-se como fontes o molibdato de amônio ( $54 \%$ de Mo) e a ureia ( $45 \%$ de $N$ ).

Procedeu-se a aplicação de Azospirillum brasilense, Rhizobium tropici e molibdênio nas sementes em doses de 4,4 g. $\mathrm{kg}^{-1}$ para ambos os produtos biológicos e $50 \mathrm{~g}$.ha-1 ${ }^{-1}$ do micronutriente. Para garantir maior adesão, permitir melhor distribuição e promover mistura mais uniforme e homogênea dos inoculantes turfosos e do molibdato de amônio às sementes, estas foram umedecidas com solução açucarada a $10 \%$

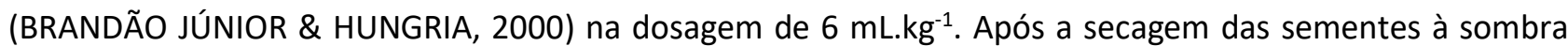
estas foram semeadas em profundidade padrão de $2 \mathrm{~cm}$. O nitrogênio na forma de ureia foi adicionado ao solo em quantidade equivalente a $30 \mathrm{~kg} \cdot \mathrm{ha}^{-1}$ e incorporado manualmente a $3 \mathrm{~cm}$ de profundidade no momento da semeadura. No estádio fenológico $V_{3}$ foi realizado o desbaste, mantendo-se apenas a planta considerada mais vigorosa por vaso. 
No período experimental as irrigações foram realizadas de forma manual e regular, visando manter a umidade do solo em torno de $70 \%$ da capacidade de campo. As condições ambientais de temperatura e umidade relativa do ar foram monitoradas no interior da casa de vegetação por meio de datalogger. A temperatura do solo foi também verificada diariamente utilizando-se termômetro tipo espeto a $14 \mathrm{~cm}$ de profundidade. As médias mínimas de temperatura e umidade do ar obtidas corresponderam a $19{ }^{\circ} \mathrm{C}$ e $31 \%$ e as máximas foram de $42{ }^{\circ} \mathrm{C}$ e $89 \%$. A temperatura média do solo foi de $34{ }^{\circ} \mathrm{C}$.

O experimento foi avaliado quando no mínimo $50 \%$ das plantas apresentavam o pendão evidente, caracterizando o estádio VT. A altura das plantas foi obtida por medição destas do colo até a inserção da folha bandeira, utilizando-se trena. O diâmetro basal do colmo foi verificado com paquímetro. Após o corte das plantas na região do colo para coleta da parte aérea (folhas, colmo e pendão) e das raízes (limpas em água corrente sobre peneira) estas foram submetidas a secagem em estufa a $65^{\circ} \mathrm{C}$ e pesagem até verificação de valores constantes de suas massas secas. A massa seca total foi obtida pelo somatório da parte aérea e radicular secas. $\mathrm{O}$ nitrogênio acumulado na parte aérea foi quantificado a partir do material seco de acordo com o método Kjeldahl descrito por Tedesco et al. (1995).

A análise estatística consistiu em verificar inicialmente a presença de dados discrepantes (GRUBBS, 1969), a normalidade dos erros (SHAPIRO \& WILK, 1965) e a homogeneidade das variâncias (BARTLETT, 1937). O teste F (SNEDECOR \& COCHRAN, 1948) foi aplicado para verificar os efeitos isolados (independentes) e/ou combinados (interação) do Azospirillum brasilense, Rhizobium tropici, molibdênio e nitrogênio nas plantas. Para as interações significativas efetuou-se o desdobramento da análise de variância para avaliar os efeitos de um fator dentro do outro.

\section{RESULTADOS E DISCUSSÃO}

O adubo nitrogenado aplicado na semeadura contribuiu para o aumento da altura, massas secas da parte aérea, raiz e total das plantas (tabela 1). Respostas positivas relacionadas ao incremento destas variáveis no milho em função da adubação nitrogenada na semeadura e/ou em cobertura foram verificadas por Carvalho et al. (2013), Morais et al. (2015) e Repke et al. (2013), evidenciando a relação direta do suprimento de nitrogênio com o crescimento vegetal.

Tabela 1: Altura de plantas (AP), massas secas da parte aérea (MSPA), raiz (MSR) e total (MST) do milho AG 1051 em função do nitrogênio aplicado no solo na semeadura

\begin{tabular}{|c|c|c|c|}
\hline \multirow{2}{*}{ Variáveis } & \multicolumn{2}{|c|}{ Nitrogênio } & \multirow{2}{*}{ CV (\%) } \\
\hline & Ausência & Presença & \\
\hline $\mathrm{AP}(\mathrm{m})$ & $1,77 \mathrm{~b}$ & $1,83 \mathrm{a}$ & 7,64 \\
\hline MSPA (g) & $109,80 \mathrm{~b}$ & $114,09 \mathrm{a}$ & 6,65 \\
\hline MSR (g) & $64,19 \mathrm{~b}$ & $70,04 \mathrm{a}$ & 17,94 \\
\hline MST (g) & $173,99 \mathrm{~b}$ & $184,12 \mathrm{a}$ & 7,40 \\
\hline
\end{tabular}

Médias seguidas de letras distintas nas linhas diferem $(p<0,05)$ entre si pelo teste $F$.

A inoculação de Azospirillum brasilense combinada à adubação nitrogenada do solo propiciou aumento de $16 \%$ na quantidade de nitrogênio acumulada na parte aérea das plantas (tabela 2). 0 resultado obtido sugere que, para esta variável, as práticas se complementaram, provavelmente em função da atividade diazotrófica da rizobactéria que pode ter contribuído para incrementar a quantidade do nitrogênio 
requerido pelo milho via fixação biológica do mesmo. O Azospirillum brasilense pode, ainda, ter melhorado o aproveitamento do elemento disponibilizado às plantas mediante adubação, uma vez que esta espécie microbiana atua na síntese de auxinas que estimulam o crescimento de raízes laterais e pelos radiculares aumentando a superfície de absorção de nutrientes presentes no solo. Respostas positivas no acúmulo de nitrogênio foliar em milho mediante o uso combinado de Azospirillum brasilense e nitrogênio já foram verificadas por Araújo et al. (2014).

Tabela 2: Nitrogênio acumulado na parte aérea (NAPA) do milho AG 1051 em função da interação entre Azospirillum brasilense e nitrogênio

\begin{tabular}{|c|c|c|c|c|}
\hline \multirow{2}{*}{ Variável } & \multirow{2}{*}{ Azospirillum brasilense } & \multicolumn{2}{|c|}{ Nitrogênio } & \multirow{2}{*}{$\mathrm{CV}(\%)$} \\
\hline & & Ausência & Presença & \\
\hline \multirow{2}{*}{ NAPA (g) } & Ausência & $874,59 \mathrm{Aa}$ & $832,12 \mathrm{Ba}$ & \multirow{2}{*}{19,35} \\
\hline & Presença & $834,09 \mathrm{Ab}$ & $967,27 \mathrm{Aa}$ & \\
\hline
\end{tabular}

Médias seguidas de letras distintas, maiúsculas na coluna e minúscula na linha, diferem $(p<0,05)$ entre si pelo teste $F$.

A altura das plantas obtida na ausência $(1,83 \mathrm{~m}$ ) de adubação molíbdica foi maior ( $p<0,05 ; C V=7,64 \%$ ) que a verificada $(1,77 \mathrm{~m})$ na presença deste micronutriente. Entretanto, embora tenha ocorrido redução desta variável, tal situação pode não representar efeito prejudicial, uma vez que, visando melhorar aspectos relacionados à diminuição do espaçamento entre plantas com o objetivo de aumentar a população destas por unidade de área, menor altura torna-se uma característica desejável para o milho e, inclusive, esta tem sido almejada em pesquisas para o melhoramento genético da espécie.

A aplicação combinada nas sementes de Azospirillum brasilense ou Rhizobium tropici e molibdênio promoveu incrementos de $63 \%$ e $60 \%$, respectivamente, na quantidade de nitrogênio acumulada na parte aérea das plantas (tabela 3). Este resultado evidencia a contribuição destas espécies microbianas para potencializar o efeito da adubação molíbdica e a deste micronutriente em otimizar o desempenho diazotrófico das rizobactérias. Além disso, a interação destes microrganismos com o molibdênio pode ter contribuído para melhorar o aproveitamento do $\mathrm{N}$ oriundo tanto da fixação biológica quanto da adubação via fertilizantes químicos. $\mathrm{O}$ aumento da quantidade de nitrogênio acumulada no milho derivado do uso de molibdênio já foi verificado quando este foi combinado à Azospirillum brasilense (GANAPATHY \& SAVALGI, 2006) e a adubo nitrogenado (VALENTINI et al., 2005).

Tabela 3: Nitrogênio acumulado na parte aérea (NAPA) do milho AG 1051 em função da interação entre Azospirillum brasilense, Rhizobium tropici e molibdênio

\begin{tabular}{|c|c|c|c|c|c|}
\hline \multirow{2}{*}{ Variável } & \multicolumn{2}{|c|}{ Rizobactérias } & \multicolumn{2}{|c|}{ Molibdênio } & \multirow{2}{*}{ CV (\%) } \\
\hline & Azospirillum brasilense & Rhizobium tropici & Ausência & Presença & \\
\hline \multirow{4}{*}{ NAPA (mg) } & Ausência & Ausência & $892,18 \mathrm{AaA}$ & $621,99 \mathrm{Bb} \underline{B}$ & \multirow{4}{*}{19,35} \\
\hline & Presença & Ausência & 927,77 Aaa & 1013,60 Aaa & \\
\hline & Ausência & Presença & $902,72 \mathrm{Aa} \underline{\mathrm{A}}$ & $996,54 \mathrm{Aa} \underline{\mathrm{A}}$ & \\
\hline & Presença & Presença & 826,46 Aaa & $834,88 \mathrm{Bab}$ & \\
\hline
\end{tabular}

Médias seguidas de letras distintas, maiúscula na coluna, minúscula na linha, maiúscula sublinhada na coluna para primeira e terceira médias e minúscula sublinhada na coluna para segunda e quarta médias, diferem $(p<0,05)$ entre si pelo teste $\mathrm{F}$.

A coinoculação de Azospirillum brasilense e Rhizobium tropici na presença de molibdênio reduziu o nitrogênio acumulado na parte aérea do milho (tabela 3). Portanto, a utilização simultânea destas espécies de microrganismos combinada ao micronutriente não foi benéfica para o acúmulo de $\mathrm{N}$ nas plantas, 
causando redução de $18 \%$ no total deste. Esta situação pode ter ocorrido devido a competição pelo nitrogênio necessário a sobrevivência dos organismos envolvidos na interação incluindo, neste caso, tanto as bactérias como as plantas.

Os resultados obtidos indicam que o molibdênio produz efeito positivo no incremento da quantidade de nitrogênio acumulada na planta somente quando está associado a apenas uma das rizobactérias (Azospirillum brasilense ou Rhizobium tropici). Além disso, quando não foi efetuada a adubação molíbdica não se verificou diferença $(p>0,05)$ entre os teores de nitrogênio do milho (tabela 3$)$, evidenciando, desta forma, que o micronutriente tem influência direta na atividade diazotrófica tanto de uma espécie microbiana quanto da outra.

A aplicação de molibdênio nas sementes, na ausência de Azospirillum brasilense e Rhizobium tropici, resultou na redução em 30\% do $\mathrm{N}$ acumulado na parte aérea do milho (tabela 3). Este resultado destaca a importância destas rizobactérias, especialmente de forma isolada, em melhorar o desempenho da adubação molíbdica em benefício da otimização do aproveitamento de nitrogênio pela planta. Embora o molibdênio seja componente básico da redutase do nitrato, apenas sua aplicação não garante necessariamente a otimização do desempenho desta enzima uma vez que este depende também de outros fatores relacionados ao solo e a planta. Portanto, para ampliar o potencial do molibdênio em aumentar a quantidade de $\mathrm{N}$ acumulada na planta é recomendável inocular nas sementes uma ou outra espécie microbiana.

Não houve efeito $(p>0,05)$ isolado e/ou combinado de Azospirillum brasilense, Rhizobium tropici, molibdênio e nitrogênio para o diâmetro basal do colmo. O resultado obtido para esta variável na época de avaliação do experimento (estádio VT) é de certa forma previsível, uma vez que neste período há tendência de estabilização do crescimento do colmo, independente dos tratamentos considerados. Picazevicz et al. (2017) observaram diferença no diâmetro de colmo em trabalho com milho avaliado no estádio V12, portanto quando este ainda estava em fase de crescimento vegetativo e, assim, com maior potencial de ser influenciado pelo efeito dos tratamentos.

\section{CONCLUSÕES}

A adubação nitrogenada na dose de $30 \mathrm{~kg} \cdot \mathrm{ha}^{-1}$ na semeadura aumenta o crescimento do milho AG 1051. A adubação molíbdica na dose de $50 \mathrm{~g} \cdot \mathrm{ha}^{-1}$ reduz a altura de plantas do milho AG 1051. A inoculação das sementes do milho AG 1051 com Azospirillum brasilense associada à adubação nitrogenada possibilita aumento da quantidade de nitrogênio acumulada na parte aérea das plantas. A inoculação das sementes do milho AG 1051 com Azospirillum brasilense ou Rhizobium tropici combinada a adubação molíbdica contribui para incrementar a quantidade de nitrogênio acumulada na parte aérea das plantas. A coinoculação das sementes do milho AG 1051 com Azospirillum brasilense e Rhizobium tropici combinada a adubação molíbdica reduz a quantidade de nitrogênio acumulada na parte aérea das plantas.

\section{REFERÊNCIAS}

AKHTAR, N.; MUJEEB, F.; QURESHI, M. A.; RAFIQUE, M.; RIAZ, A.; ALI, M. A. Phosphate solubilizing potential of
Rhizobium and Bacillus species for enhancing yield and available phosphorus in maize crop (Zea mays). 
International Journal of Agronomy and Agricultural

Research, Dhaka, v.4, n.1, p.58-66, 2014. DOI:

https://doi.org/10.6084/M9. FIGSHARE.1538550

ARAÚJO, R. M.; ARAÚJO, A. S. F.; NUNES, L. A. P. L.; FIGUEIREDO, M. V. B. Resposta do milho verde a inoculação com Azospirillum brasilense e níveis de nitrogênio. Ciência Rural, Santa Maria, v.44, n.9, p.1556-1560, 2014. DOI: https://doi.org/10.1590/0103-8478cr20130355

BARTLETT, M. S. Properties of sufficiency and statistical tests. Proceedings of the Royal Society of London, London, v.160, n.901, p.268-282, 1937. DOI:

https://doi.org/10.1098/rspa.1937.0109

BRANDÃO JÚNIOR, O.; HUNGRIA, M. Efeito de concentrações de solução açucarada na aderência do inoculante turfoso às sementes, na nodulação e no rendimento da soja. Revista Brasileira de Ciência do Solo, Viçosa, v.24, n.3, p.515-526, 2000. DOI: http://dx.doi.org/10.1590/S0100-06832000000300005

CARVALHO, E. V.; CANCELLIER, L. L.; AFFÉRRI, F. S.; DOTTO, M. A.; PEUZIO, J. M.; CRUZ, O. S. Crescimento de milho em níveis contrastantes de nitrogênio e sua correlação com produtividade de grãos. Revista Brasileira de Ciências Agrárias, Recife, v.8, n.3, p.351-357, 2013. DOI: https://doi.org/10.5039/agraria.v8i3a2066

COSTA, R. R. G. F.; QUIRINO, G. S. F.; NAVES, D. C. F.; SANTOS, C. B.; ROCHA, A. F. S. Efficiency of inoculant with Azospirillum brasilense on the growth and yield of secondharvest maize. Pesquisa Agropecuária Tropical, Goiânia, v.45, n.3, p.304-311, 2015. DOI:

https://doi.org/10.1590/1983-40632015v4534593

DARTORA, J.; GUIMARÃES, V. F.; MENEZES, C. R. J.; FREIBERGER, M. B.; CASTOLDI, G.; GONÇALVES, E. D. V. Maize response to inoculation with strains of plant growthpromoting bacteria. Revista Brasileira de Engenharia Agrícola e Ambiental, Campina Grande, v.20, n.7, p.606-611, 2016. DOI: https://doi.org/10.1590/18071929/agriambi.v20n7p606-611

EMBRAPA. Empresa Brasileira de Pesquisa Agropecuária. Manual de métodos de análise de solo. 2 ed. Rio de Janeiro: Embrapa, 1997.

GANAPATHY, B. A.; SAVALGI, V. P. Effect of micronutrients on the performance of Azospirillum brasilense on the nutrient uptake, growth and yield in maize crop. Karnataka Journal of Agricultural Sciences, Krishnagar, v.19, n.1, p.6670, 2006.

GRUBBS, F. E. Procedures for detecting outlying observations in samples. Technometrics, Princeton, v.11, n.1, p.1-21, 1969. DOI:

https://doi.org/10.1080/00401706.196910490657

MAZZUCHELLI, R. C. L.; SOSSAI, B. F.; ARAÚJO, F. F. Inoculação de Bacillus subtilis e Azospirillum brasilense na cultura do milho. Coloquium Agrariae, Presidente Prudente, v.10, n.2, p.40-47, 2014. DOI:

http://doi.org10.5747/ca.2014.v10.n2.a106

MORAIS, T. P.; BRITO, C. H.; BRANDÃO, A. M.; REZENDE, W. $S$. Inoculation of maize with Azospirillum brasilense in the seed furrow. Ciência Agronômica, Fortaleza, v.47, n.2 p.290-298, 2016. DOI: https://doi.org/10.5935/18066690.20160034

MORAIS, T. P.; BRITO, C. H.; FERREIRA, A. S.; LUZ, J. M. Q. Aspectos morfofisiológicos de plantas de milho e bioquímico do solo em resposta à adubação nitrogenada e à inoculação com Azospirillum brasilense. Ceres, Viçosa, v.62, n.6, p.507509, 2015. DOI: http://dx.doi.org/10.1590/0034$\underline{737 \times 201562060012}$

NOGUEIRA, M. A.; HUNGRIA, M. Coinoculação: técnica favorece a soja e o feijão. Campo \& Negócios, Uberlândia, v.9, n.127, p.52-53, 2013.

OSÓRIO FILHO, B. D.; BINZ, A.; LIMA, R. F.; GIONGO, A.; SÁ, E. L. S. Promoção de crescimento de arroz por rizóbios em diferentes níveis de adubação nitrogenada. Ciência Rural, Santa Maria, v.46, n.3, p.478-485, 2016.

PICAZEVICZ, A. A. C.; KUSDRA, J. F.; MORENO, A. L. Maize growth in response to Azospirillum brasilense, Rhizobium tropici, molybdenum and nitrogen. Revista Brasileira de Engenharia Agrícola e Ambiental, Campina Grande, v.21, n.9, p.623-627, 2017. DOI: http://doi.org/10.1590/18071929/agriambi.v21n9p623-627

QUADROS, P. D.; ROESCH, L. F. W.; SILVA, P. R. F.; VIEIRA, V. M.; ROEHRS, D. D.; CAMARGO, F. A. O. Desempenho agronômico a campo de híbridos de milho inoculados com Azospirillum. Ceres, Viçosa, v.61, n.2, p.209-218, 2014. DOI: http://doi.org/10.1590/S0034-737X2014000200008

REPKE, R. A.; CRUZ, S. J. S.; SILVA, C. J.; FIGUEIREDO, P. G.; BICUDO, S. J. Eficiência da Azospirillum brasilense combinada com doses de nitrogênio no desenvolvimento de plantas de milho. Revista Brasileira de Milho e Sorgo, Sete Lagoas, v.12, n.3, p.214-226, 2013. DOI: http://doi.org/10.18512/19806477/rbms.v12n3p214-226

SHAPIRO, S. S.; WILK, M. B. An analysis of variance test for normality (complete samples). Biometrika, Boston, v.52, n.4, p.591-611, 1965. DOI: https://doi.org/10.2307/2333709

SINGH, R. K.; MALIK, N.; SINGH, S. Impact of rhizobial inoculation and nitrogen utilization in plant growth promotion of maize (Zea mays L.). Nusantara Bioscience, Surakarta, v.5, n.1, p.8-14, 2013. DOI:

https://doi.org/10.13057/nusbiosci/n050102

SNEDECOR, G. W.; COCHRAN, W. G. Statistical methods. Ames: lowa State University Press, 1948.

TAN, K. Z.; RADZIAH, O.; HALIMI, M. S.; KHAIRUDDIN, A. R.; HABIB, S. H.; SHAMSUDDIN, Z. H. Isolation and characterization of rhizobia and plant growth-promoting rhizobacteria and their effects on growth of rice seedlings. American Journal of Agricultural and Biological Science, New York, v.9, n.3, p.342-360, 2014. DOI: https://doi.org/10.3844/ajabssp.2014.342.360

TEDESCO, M. J.; GIANELLO, C.; BISSANI, C. A.; BOHNEN, H.; VOLKWEISS, S. J. Análise de solo, plantas e outros materiais. 2 ed. Porto Alegre: EDUFRGS, 1995.

TORTORA, G. J.; FUNKE, B. R.; CASE, C. L. Microbiologia. 12 ed. Porto Alegre: Artmed, 2017. 
VALENTINI, L.; COELHO, F. C.; FERREIRA, M. dos S. Teor de

(Zea mays L.) submetidos às adubações nitrogenada $\mathrm{e}$ nitrogênio foliar e produtividade de três cultivares de milho molíbdica. Ceres, Viçosa, v.52, n.302, p.567-577, 2005.

A CBPC - Companhia Brasileira de Produção Científica (CNPJ: 11.221.422/0001-03) detém os direitos materiais desta publicação. Os direitos referem-se à publicação do trabalho em qualquer parte do mundo, incluindo os direitos às renovações, expansões e disseminações da contribuição, bem como outros direitos subsidiários. Todos os trabalhos publicados eletronicamente poderão posteriormente ser publicados em coletâneas impressas sob coordenação da Sustenere Publishing, da Companhia Brasileira de Produção Científica e seus parceiros autorizados. Os (as) autores (as) preservam os direitos autorais, mas não têm permissão para a publicação da contribuição em outro meio, impresso ou digital, em português ou em tradução. 